\title{
Electronic and Magnetic Properties of One Dimensional Sandwich Transition Metal-Anthracene Molecular Wires
}

\author{
Guang Yang ${ }^{1}$, Huiyang Zhang ${ }^{1}$, Yijun Yang ${ }^{1}$, Yudi Wang ${ }^{1}$, Xinzi Xv ${ }^{1}$, Xinli Zhao ${ }^{1}$, Lijuan \\ Meng $^{2}$, Xiaojing $\mathrm{Yao}^{3}$, Xiuyun Zhang $^{1}$, and Yongjun Liu ${ }^{1}$
}

${ }^{1}$ Yangzhou University

${ }^{2}$ Yancheng Institute of Technology

${ }^{3}$ Hebei Normal University

September 25, 2021

\begin{abstract}
Organometallic sandwich complexes have been attracting tremendous interest for their potential applications in electronics and spintronics. Here, we systematically studied the structures, electronic and magnetic properties of one dimensional (1D) transition metal (TM)-anthracene (Ant) sandwich molecular wires (SMWs), [TM2Ant][?] and [TM3Ant][?] (TM=Ti, V, Cr, $\mathrm{Mn}$ ), based on density functional theory calculations. Our results showed that all the 1D SMWs display normal sandwich configurations with their binding energies closely related to the choice of TM atoms. Excepting 1D [Mn2Ant][?] and [Fe3Ant][?] favoring antiferromagnetic ordering, most 1D [TM2Ant][?] and [TM3Ant][?] SMWs display robust ferromagnetic feathers. Particularly, 1D [Cr3Ant][?] SMW is revealed to be ferromagnetic half-metal with large magnetic moment of $28.0 \mu \mathrm{B}$ per unit cell. Further spintransport calculations double proved that 1D [Cr3Ant][?] SMW are good spintransport molecular devices. Our findings shed light on the properties of 1D Ant based SMWs and propose a new way to design potential electronic and spintronic devices.
\end{abstract}

\section{Hosted file}

manuscript.docx available at https://authorea.com/users/436334/articles/538726-electronicand-magnetic-properties-of-one-dimensional-sandwich-transition-metal-anthracenemolecular-wires 